\title{
miR-17-5p regulates the proliferation and apoptosis of human trabecular meshwork cells by targeting phosphatase and tensin homolog
}

\author{
XIAOYUAN WANG ${ }^{1}$, ZHIJIAN LI $^{1}, \mathrm{JIE} \mathrm{BAI}^{1}, \mathrm{WUQI} \mathrm{SONG}^{2}$ and FENGMIN ZHANG ${ }^{2}$ \\ ${ }^{1}$ Department of Ophthalmology, The First Affiliated Hospital of Harbin Medical University; \\ ${ }^{2}$ Department of Microbiology, Harbin Medical University, Heilongjiang Provincial Key Laboratory of Infection and \\ Immunity, Key Laboratory of Pathogenic Biology, Harbin, Heilongjiang 150086, P.R. China
}

Received July 12, 2017; Accepted January 17, 2019

DOI: $10.3892 / \mathrm{mmr} .2019 .9973$

\begin{abstract}
Glaucoma is one of the leading causes of blindness. Previous studies have indicated that the oxidative stress-induced apoptosis of trabecular meshwork cells (TMCs) may serve a key role in the pathogenesis of glaucoma, and that micro RNA(miR)-17-5p may be involved in this process. However, the specific mechanisms require further investigation. The aim of the present study was to investigate the effects of miR-17-5p on the proliferation and apoptosis of human TMCs (HTMCs) in response to oxidative stress. It was observed that exposure to $\mathrm{H}_{2} \mathrm{O}_{2}$ induced a significant decrease in the proliferation and a marked increase in the apoptosis of HTMCs. $\mathrm{H}_{2} \mathrm{O}_{2}$ exposure also suppressed the expression of miR-17-5p and promoted the expression of phosphatase and tensin homolog (PTEN). Furthermore, transient overexpression of miR-17-5p induced a significant increase in the proliferation and a significant decrease in the apoptosis of HTMCs by affecting the expression of PTEN, and the apoptosis-related proteins B-cell lymphoma-associated X protein (Bax), B-cell lymphoma-extra large (Bcl-xL) and B-cell lymphoma-2 (Bcl-2). However, knockdown of miR-17-5p demonstrated the opposite results. The results of a dual luciferase reporter assay demonstrated that PTEN may be a direct target of miR-17-5p. In conclusion, miR-17-5p was downregulated in HTMCs under oxidative conditions, and miR-17-5p may regulate the apoptosis of HTMCs by targeting PTEN. These results provide a novel theoretical basis and potential therapeutic target for the treatment of glaucoma.
\end{abstract}

Correspondence to: Dr Fengmin Zhang, Department of Microbiology, Harbin Medical University, Heilongjiang Provincial Key Laboratory of Infection and Immunity, Key Laboratory of Pathogen Biology, 157 Baojian Road, Harbin, Heilongjiang 150086, P.R. China

E-mail: dr_fengmingzhang@outlook.com

Key words: glaucoma, micro RNA-17-5p, phosphatase and tensin homolog, human trabecular meshwork cells, apoptosis

\section{Introduction}

Glaucoma is a common ophthalmic disease, and it is the second leading cause of blindness worldwide (1). It is estimated that by 2020 , the number of patients with glaucoma will reach approximately 80 million (2). Glaucoma can be divided into open-angle glaucoma (OAG) and angle-closure glaucoma (ACG), and in Western countries, approximately $75 \%$ of glaucoma patients are diagnosed with OAG (3), while in Asian countries, ACG is more prevalent than OAG (4). In 2010, 86.5\% of ACG patients were resident in Asia, and the largest number of patients affected by ACG was in China (47.5\% of the total) (5). In recent years, increasing evidence has indicated that the oxidative stress-induced senescence of trabecular meshwork cells (TMCs) may play a key role in the occurrence and development of glaucoma $(6,7)$; however, the specific mechanisms still require further investigation.

MicroRNAs (miRNAs) are short non-coding RNAs (length, 22 nucleotides) that can bind to the 3'-UTR of target mRNAs and consequently suppress the expression of target genes. miRNAs can regulate a variety of biological activities, including embryonic development, angiogenesis, cell differentiation, proliferation and apoptosis (8-10). With the development of next-generation sequencing and bioinformatics methods, differentially expressed miRNAs that are potentially involved in the pathogenesis of diseases, including cancers $(11,12)$ and glaucoma $(13-15)$, have been successfully identified. The targets of these miRNAs, as well as related signaling pathways, have also been predicted. miR-17-5p is located on human chromosome $13 \mathrm{q} 31$, and it belongs to the miR-17-92 cluster (16). miR-17-5p is up-regulated in many tumor types and participates in the pathogenesis of different cancers as an oncogene (17-19). Besides cancer-related studies, miR-17-5p has been reported to promote oxidative stress-induced apoptosis of cardiomyocytes in ischemia/reperfusion-induced cardiac injury animal models (20). It has also been observed that knockdown of miR-17-5p can restore the expression of the very-low-density lipoprotein receptor and alleviate the symptoms of atherosclerosis in ApoE-/mouse models (21). It has been observed that miR-17-5p is down-regulated in the trabecular meshwork under oxidative 
stress (22); however, the specific mechanisms have not yet been investigated.

In the present study, we focused on the roles of miR-17-5p in glaucoma and its related mechanisms. Human TMCs (HTMCs) were treated with $\mathrm{H}_{2} \mathrm{O}_{2}$ to induce oxidative stress, and the effects of miR-17-5p on the behaviors of HTMCs under oxidative stress were explored. We hypothesized that miR-17-5p would regulate the proliferation and apoptosis of HTMCs by targeting phosphatase and tensin homolog (PTEN). The present study may provide a theoretical basis and novel therapeutic target for the treatment of glaucoma.

\section{Materials and methods}

HTMC culture. HTMCs were purchased from Sciencell Research Laboratories (San Diego, CA, USA). According to the product information, HTMCs were isolated from the juxtacanalicular and corneoscleral regions of human eyes, and characterized by immunofluorescence methods using $\alpha$-smooth muscle actin- and fibronectin-specific antibodies. Cells were cultured in a humidified incubator at $37^{\circ} \mathrm{C}$ with $5 \% \mathrm{CO}_{2}$ using Dulbecco's modified Eagle's medium (DMEM; Gibco; Thermo Fisher Scientific, Inc., Waltham, MA, USA) supplied with $10 \%$ fetal bovine serum (Gibco; Thermo Fisher Scientific, Inc.) and $1 \%$ penicillin-streptomycin solution (Gibco; Thermo Fisher Scientific, Inc.). Oxidative stress was induced by treating HTMCs with DMEM that contained $300 \mu \mathrm{M} \mathrm{H}_{2} \mathrm{O}_{2}$ (Beyotime Institute of Biotechnology, Shanghai, China) for 3-7 h. Then, the cells were harvested for future analysis.

Cell transfection. The miR-17-5p inhibitor and miR-17-5p mimic oligonucleotides were synthesized by GenePharma Co., Ltd., (Shanghai, China). HTMCs were cultured until confluence, trypsinized and seeded onto new six-well plates at 100,000 cells/well. Cells were then transfected with either miR-17-5p inhibitor or miR-17-5p mimic using Lipofectamine ${ }^{\circledR} 3000$ reagent (Invitrogen; Thermo Fisher Scientific, Inc.), according to the manufacturer's protocol. After transfection, cells were cultured for $48 \mathrm{~h}$ and harvested for future analysis.

Cell proliferation analysis. An MTT assay was performed to determine the viability of HTMCs following different treatments. An MTT proliferation assay kit (Sigma-Aldrich; Merck KGaA, Darmstadt, Germany) was used, according to the manufacturer's protocol.

Cellular apoptosis analysis. For cellular apoptosis analysis, HTMCs subjected to different treatments were stained using an Annexin V-FITC apoptosis detection kit (Sigma-Aldrich; Merck KGaA). The apoptosis rate of the cells in different groups was detected and analyzed using a BD FACSVerse flow cytometer (BD Biosciences, San Jose, CA, USA), according to the manufacturer's protocol.

Reverse transcription-quantitative PCR (RT-qPCR). Total RNA was extracted from HTMCs using TRIzol (Invitrogen; Thermo Fisher Scientific, Inc.), and reverse transcribed into cDNA using the PrimeScript ${ }^{\mathrm{TM}}$ RT Master Mix (Perfect Real
Time) kit (Takara Biotechnology Co., Ltd., Dalian, China), then qPCR was performed using the SYBR ExScript RT-PCR kit (Takara Biotechnology Co., Ltd.) on an ABI 7300 Real-Time PCR System (Applied Biosystems; Thermo Fisher Scientific, Inc.), according to the manufacturer's protocol. The thermocycling profiles are shown in Table I. The relative expression of PTEN in each sample was normalized to the level of GAPDH using the $2^{-\Delta \Delta \mathrm{Cq}}$ method. The expression of miR-17-5p was examined using the Hairpin-it ${ }^{\mathrm{TM}}$ miRNAs qPCR Quantitation Kit (GenePharma Co., Ltd.) according to the manufacturer's protocol, and U6 (RNU6B; GenePharma Co., Ltd.) was used for normalization. Primers were synthesized by GenScript (Nanjing, China), and the sequences of the primers are shown in Table II.

Western blot analysis. Cells were lysed using RIPA buffer (Beyotime Institute of Biotechnology), and the concentration of total protein was measured using a BCA Kit (Beyotime Institute of Biotechnology). Next, SDS-PAGE was performed to separate the proteins, and the proteins were then transferred onto polyvinylidene fluoride membranes (EMD Millipore, Billerica, MA, USA). The membranes were blocked with 5\% non-fat milk and incubated with primary antibodies (anti-Bax, anti-PTEN, anti-Bcl-2, anti-Bcl-xL and anti-GAPDH, all purchased from Abcam, Cambridge, MA, USA) overnight at $4^{\circ} \mathrm{C}$. The following day, the membranes were incubated with HRP-conjugated secondary antibodies (purchased from Abcam, Cambridge, MA, USA), then washed and incubated with the enhanced chemiluminescence reagent (Beyotime Institute of Biotechnology). Finally, the signals were detected using the ChemiDoc ${ }^{\mathrm{TM}} \mathrm{XRS}+$ imaging system (Bio-Rad Laboratories, Inc., Hercules, CA, USA).

Immunocytochemistry. HTMCs were fixed in 4\% paraformaldehyde and permeabilized with $0.1 \%$ Triton $\mathrm{X}-100$, then incubated with the anti-PTEN primary antibodies for $30 \mathrm{~min}$. Cells were incubated with Alexa Fluor 488-conjugated secondary antibodies, then visualized using an Olympus fluorescent microscope (Olympus Corporation, Tokyo, Japan).

Computational methods. The target genes of miR-17-5p were predicted using online bioinformatics tools, TargetScan (http://www.targetscan.org) and miRanda (http://www. microrna.org).

Dualluciferasereporterassay.Fragments of eitherthe wild-type PTEN 3'-UTR (PTEN-3'UTR) or mutant PTEN 3'-UTR (PTEN-MUT) region that contains the miR-17-5p binding site were synthesized and cloned into the pGL6-TA-reporter plasmid (Beyotime Institute of Biotechnology). The plasmids were transfected into 293 cells using Lipofectamine ${ }^{\circledR} 3000$ for $48 \mathrm{~h}$, and the activity of the luciferase in each group was examined using the dual-luciferase reporter system (Beyotime Institute of Biotechnology), according to the manufacturer's protocol.

Statistics. All statistical analyses were conducted using SPSS v.22.0 software (IBM Corp., Armonk, NY, USA). Data are presented as the mean \pm standard deviation. Two independent sample t-tests were performed for comparisons between two 
Table I. Thermocycling profiles for reverse transcriptionquantitative polymerase chain reaction.

\begin{tabular}{lcr}
\hline Cycle no. & Temperature & Time \\
\hline 1 & $95^{\circ} \mathrm{C}$ & $30 \mathrm{Sec}$ \\
40 & $95^{\circ} \mathrm{C}$ & $5 \mathrm{Sec}$ \\
& $60^{\circ} \mathrm{C}$ & $4 \mathrm{Sec}$ \\
\hline
\end{tabular}

Table II. Sequences of the primers used in the present study.

\begin{tabular}{ll}
\hline Gene name & \multicolumn{1}{c}{ Sequences of the primer } \\
\hline miR-17-5p & F: 5'-TCTAGATCCCGAGGACTG-3' \\
& R: 5'-ATCGTGACCTGAACC-3' \\
U6 & F: 5'-CTCGCTTTGGCAGCACA-3' \\
& R: 5'-AACGCTTCACGAATTGCGT-3' \\
PTEN & F: 5'-CGACGGGAAGACAAGTTCAT-3' \\
& R: 5'-AGGTTTCCTCTGGTCCTGGT-3' \\
GAPDH & F: 5'-GACAGTCAGCCGCATCTTCT-3' \\
& R:5'-TTAAAAGCAGCCCTGGTGAC-3'
\end{tabular}

PTEN, phosphatase and tensin homolog; F, forward; R, reverse.

groups. One-way analysis of variance followed by Dunnett's post-hoc test was performed for comparisons among multiple groups. $\mathrm{P}<0.05$ was considered to indicate a statistically significant difference.

\section{Results}

Effect of oxidative stress on the proliferation and apoptosis of HTMCs in vitro. First, we investigated the effect of $\mathrm{H}_{2} \mathrm{O}_{2}$ treatment on the proliferation and apoptosis of HTMCs using MTT and flow cytometry methods. It was observed that the cell viability was significantly decreased in cells treated with $\mathrm{H}_{2} \mathrm{O}_{2}$ compared with the control group at 3,5 and $7 \mathrm{~h}(\mathrm{P}<0.01$; Fig. 1A). Moreover, $\mathrm{H}_{2} \mathrm{O}_{2}$ treatment also induced a continuous increase in the apoptosis rate of HTMCs from 0 to $7 \mathrm{~h}(\mathrm{P}<0.05$; Fig. 1B and C).

miR-17-5p was down-regulated in HTMCs under oxidative stress in vitro. In order to explore the roles of miR-17-5p in HTMCs under oxidative stress, we compared the expression of miR-17-5p in control and $\mathrm{H}_{2} \mathrm{O}_{2}$ groups at different time points using RT-qPCR methods. As shown in Fig. 2A, $\mathrm{H}_{2} \mathrm{O}_{2}$ induced a significant decrease in the expression of miR-17-5p at 5 and 7 h $(\mathrm{P}<0.05$; Fig. 2A).

$m i R-17-5 p$ can regulate the proliferation and apoptosis of HTMCs in vitro. HTMCs were transfected with either miR-17-5p mimics or miR-17-5p inhibitor, and the effect of miR-17-5p on the proliferation and apoptosis of HTMCs was examined using MTT and flow cytometry methods. As shown in Fig. 2B, the expression of miR-17-5p was significantly increased in miR-17-5p mimic-transfected HTMCs and markedly decreased
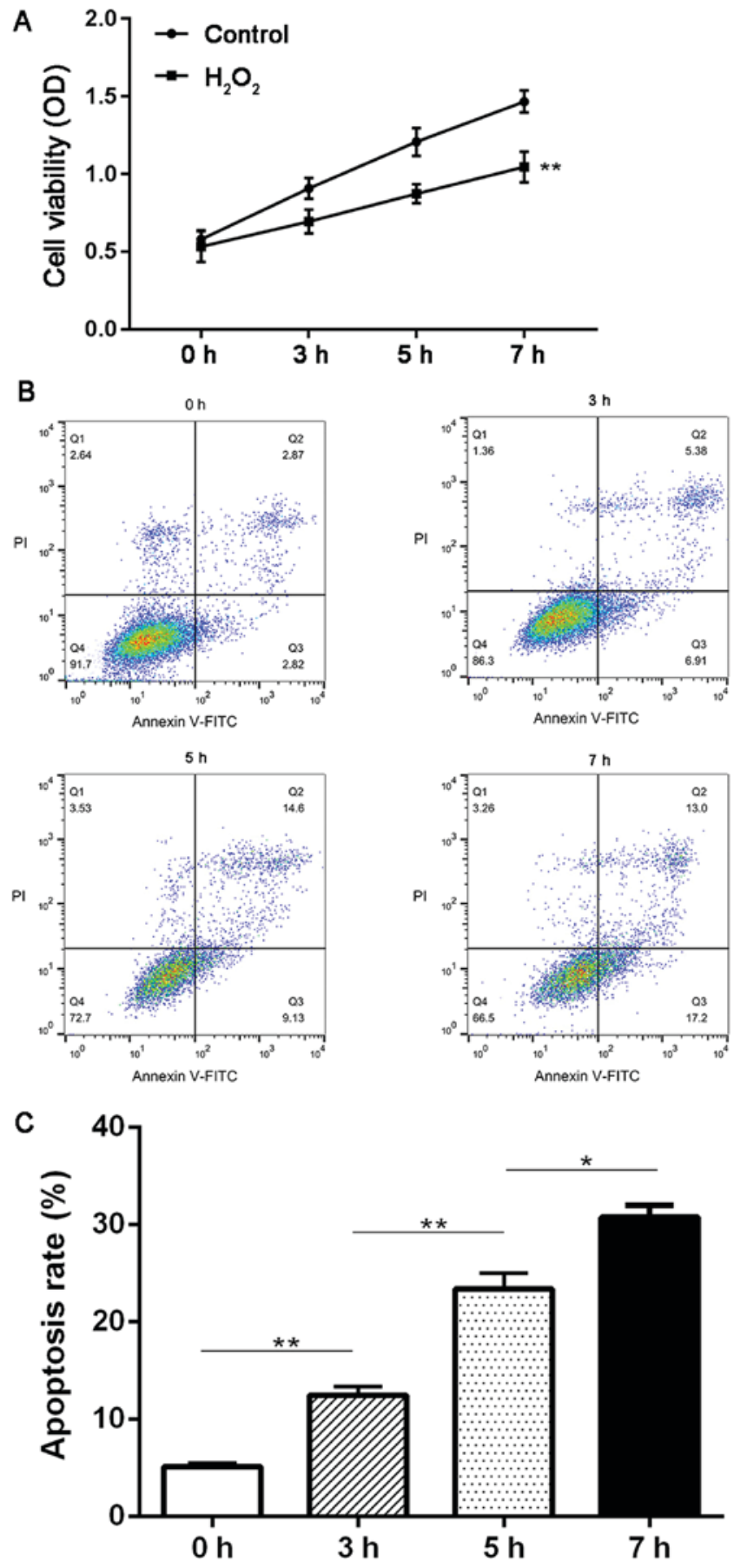

Figure 1. Effect of oxidative stress on the proliferation and apoptosis of HTMCs in vitro. (A) Proliferation rate of HTMCs in different groups by MTT assay, ${ }^{* *} \mathrm{P}<0.01$ vs. the control group; (B) Apoptosis rate of HTMCs in different groups by flow cytometry method. (C) Quantification of results in (B). ${ }^{* *} \mathrm{P}<0.01$ vs. the $3 \mathrm{~h}$ group, ${ }^{*} \mathrm{P}<0.05$ vs. the $5 \mathrm{~h}$ group.

in miR-17-5p inhibitor-transfected HTMCs, suggesting that transfection had been successfully performed. Moreover, transient overexpression of miR-17-5p induced a significant increase in the proliferation $(\mathrm{P}<0.01$; Fig. $3 \mathrm{~A}$ and $\mathrm{B})$ and a significant decrease in the apoptosis of HTMCs $(\mathrm{P}<0.01$; Fig. $3 \mathrm{C})$. Knockdown of miR-17-5p exhibited the opposite results. In addition, transfection of miR-17-5p mimics also induced a significant increase in the expression of Bcl-2 and Bcl-xL, and a marked decrease in the expression of Bax, while knockdown of miR-17-5p exhibited the opposite results (Fig. 4). 
A

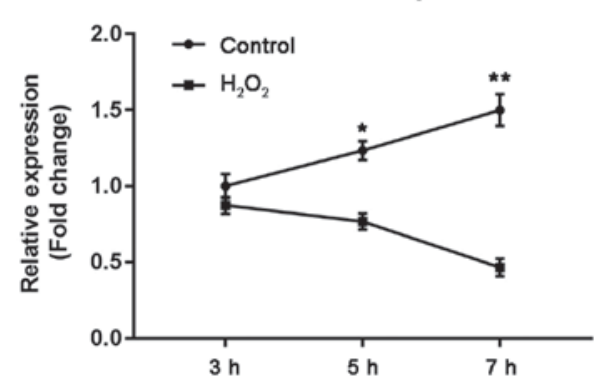

B

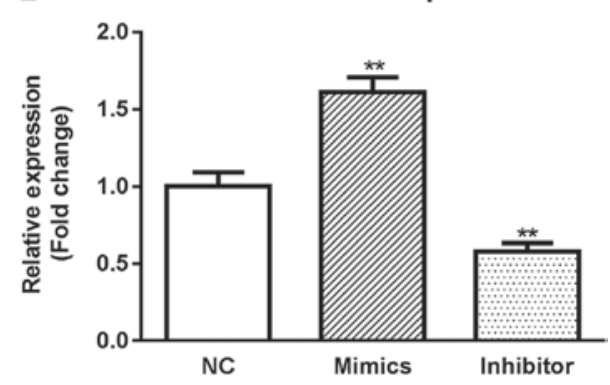

Figure 2. Expressions of miR-17-5p in HTMCs by RT-qPCR methods. (A) Effect of oxidative stress on the expression of miR-17-5p in HTMCs. (B) Effect of transfection of miR-17-5p mimics or inhibitor on the expression of miR-17-5p in HTMCs. ${ }^{*} \mathrm{P}<0.05$ and ${ }^{* *} \mathrm{P}<0.01$ vs. the control group. HTMCs, human trabecular meshwork cells.
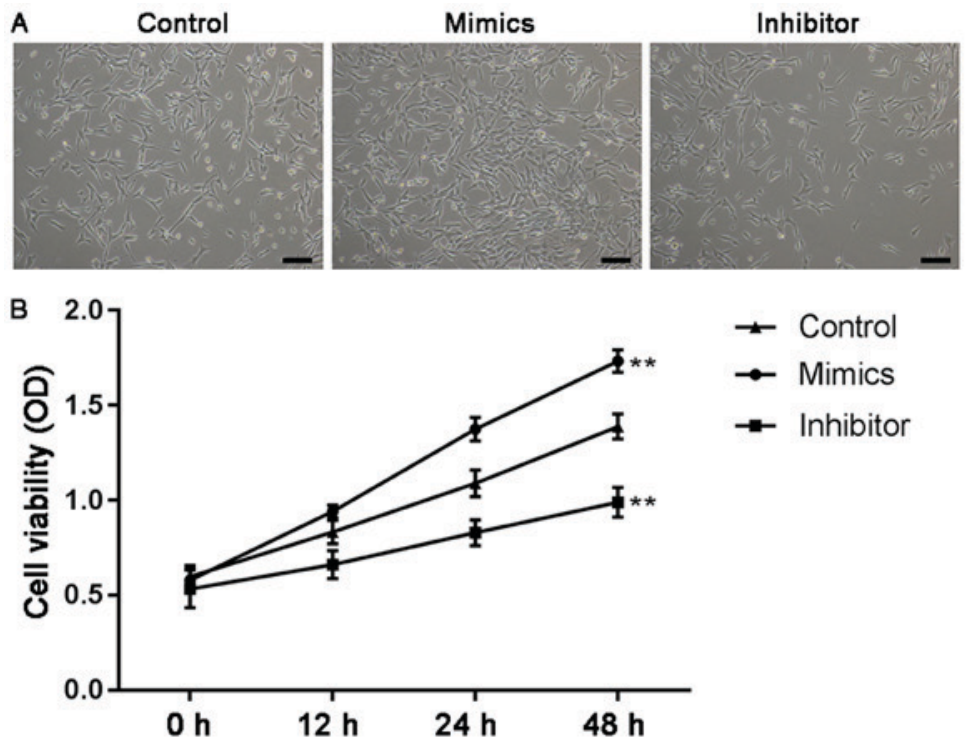

C
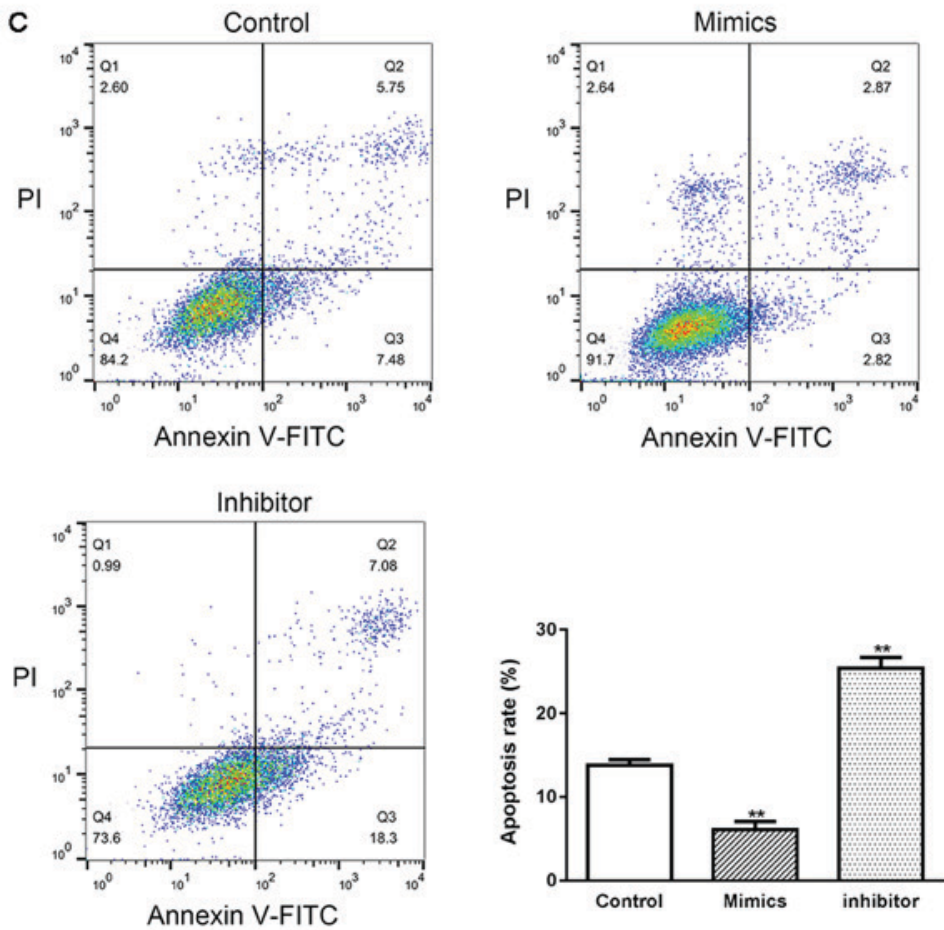

Figure 3. Effect of miR-17-5p on the proliferation and apoptosis of HTMCs in vitro. (A) Morphological feature of HTMCs in different groups by inverted microscope (scale bar, $100 \mu \mathrm{m}$ ); (B) Proliferation rate of HTMCs in different groups by MTT assay; (C) Apoptosis rate of HTMCs in different groups by flow cytometry method. ${ }^{* *} \mathrm{P}<0.01$ vs. the control group. Control, un-transfected cells; Inhibitor, miR-17-5p inhibitor transfected cells; Mimics, miR-17-5p mimics transfected cells. HTMCs, human trabecular meshwork cells. 


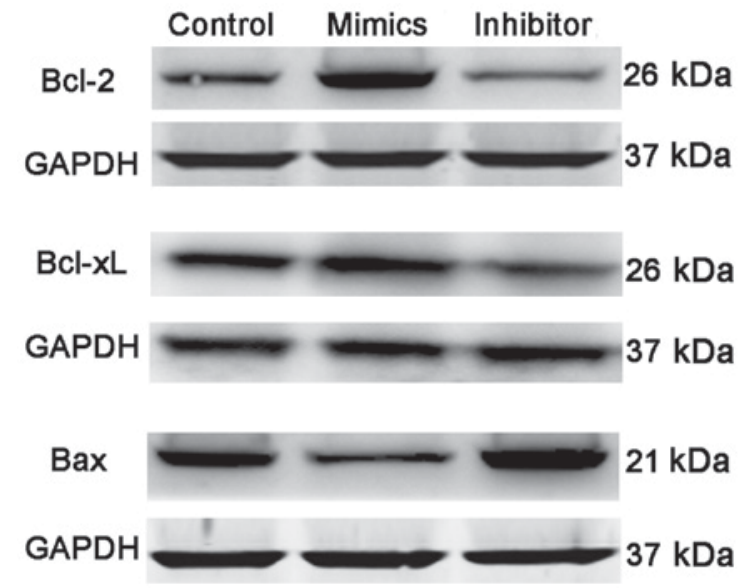

Figure 4. Effect of miR-17-5p on the expression of Bcl-2, Bcl-xL and Bax in HTMCs by western blot methods. Control, un-transfected cells; Inhibitor, miR-17-5p inhibitor transfected cells; Mimics, miR-17-5p mimics transfected cells. Bcl-2, B-cell lymphoma-2; Bcl-xL, B-cell lymphoma extra-large; BAX, Bcl-2-associated X protein; HTMCs, human trabecular meshwork cells; GAPDH, glyceraldehyde-3-phosphate dehydrogenase.

PTEN is a direct target of miR-17-5p in HTMCs. Using online bioinformatics tools, PTEN was predicted to be a direct target of miR-17-5p. Using western blot analysis, it was observed that the expression of PTEN continuously increased from 3 to $7 \mathrm{~h}$ under oxidative stress (Fig. 5A), suggesting that increased PTEN may be associated with the pathogenesis of glaucoma. Moreover, transient overexpression of miR-17-5p in HTMCs induced a significant decrease in the expression of PTEN, while knockdown of miR-17-5p promoted the expression of PTEN in HTMCs (Fig. 5B and C). Finally, the results from the dual luciferase reporter assay indicated that co-transfection of miR-17-5p mimics and wild-type PTEN-3'UTR plasmids significantly decreased the activity of the luciferases. Co-transfection of miR-17-5p mimic and PTEN-MUT exhibited no effect on the activity of the luciferases (Fig. 6), suggesting that PTEN is a direct target of miR-17-5p.

\section{Discussion}

Glaucoma is a common ophthalmic disorder that can cause damage to the optic nerve and loss of vision. Increasing evidence has indicated that oxidative stress-induced senescence of HTMCs is involved in the pathogenesis of glaucoma (7). $\mathrm{H}_{2} \mathrm{O}_{2}$ is an important oxidant in the human eye. It has been demonstrated that oxidative levels significantly increase in the aqueous humor of patients with glaucoma, which means that TMCs will also be exposed to high concentrations of $\mathrm{H}_{2} \mathrm{O}_{2}$. Thus, in previous studies, a high concentration of $\mathrm{H}_{2} \mathrm{O}_{2}$ has been used to induce oxidative stress in HTMCs, and this has now become a widely applied in vitro model in glaucoma-related studies $(23,24)$.

In recent years, the effects of miRNAs on TMCs have been discussed in many studies $(25,26)$. Li et al $(22)$, performed miRNA array analysis to examine alterations in the expression levels of microRNAs in oxidative stress-induced cellular senescence in HTMCs. It was observed that the expression of miR-17-5p was significantly decreased by treatment with a high concentration of $\mathrm{H}_{2} \mathrm{O}_{2}$. However, the specific mechanisms
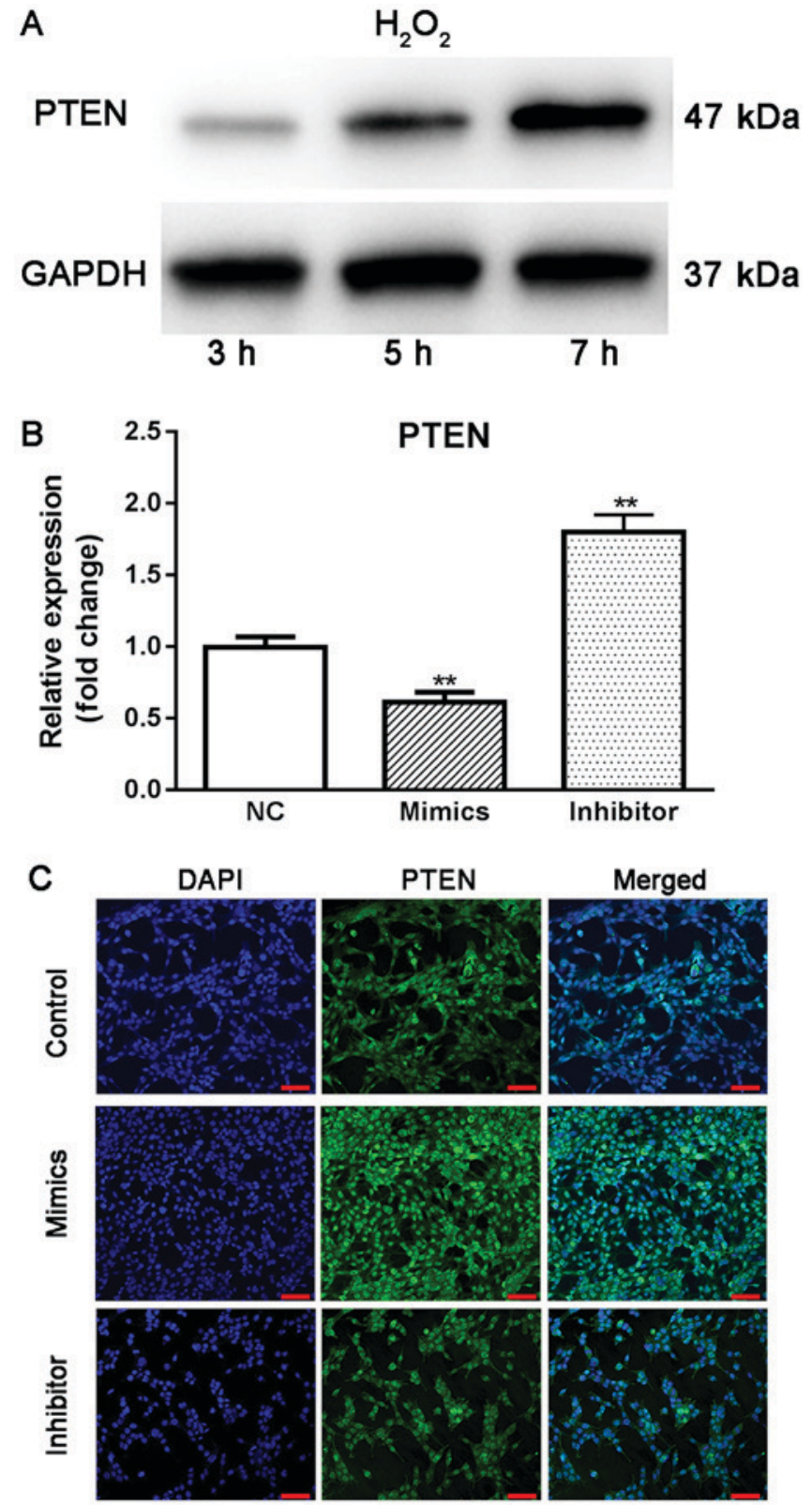

Figure 5. Roles of PTEN in the pathogenesis of glaucoma. (A) Expression of PTEN in HTMCs under oxidative stress by western blot method; (B) Effect of overexpression and knockdown of miR-17-5p on the mRNA expression of PTEN in HTMCs by RT-qPCR method; (C) Effect of overexpression and knockdown of miR-17-5p on the protein expression of PTEN in HTMCs using ICC method (scale bar, $100 \mu \mathrm{m}$ ). ${ }^{* *} \mathrm{P}<0.01$ vs. NC. NC, un-transfected cells; Inhibitor, miR-17-5p inhibitor transfected cells; Mimics, miR-17-5p mimics transfected cells. PTEN, Phosphatase and tensin homolog; HTMCs, human trabecular meshwork cells; GAPDH, glyceraldehyde-3-phosphate dehydrogenase.

underlying this remain unclear. In the present study, we identified that compared with untreated cells, $\mathrm{H}_{2} \mathrm{O}_{2}$ treatment induced a significant decrease in the proliferation and a marked increase in the apoptosis of HTMCs at each time point examined $(\mathrm{P}<0.05)$. Moreover, $\mathrm{H}_{2} \mathrm{O}_{2}$ treatment also induced a significant decrease in the expression of miR-17-5p, which was consistent with the finding from Li et al. These results indicate that miR-17-5p is down-regulated in HTMCs under oxidative stress, and miR-17-5p may participate in the mechanism of $\mathrm{H}_{2} \mathrm{O}_{2}$-induced increase in apoptosis and decrease in the proliferation of HTMCs. 


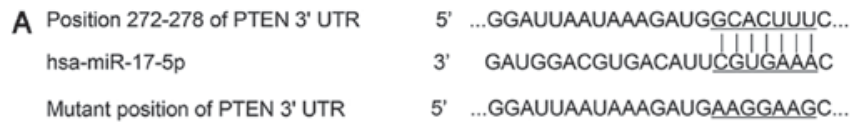

B

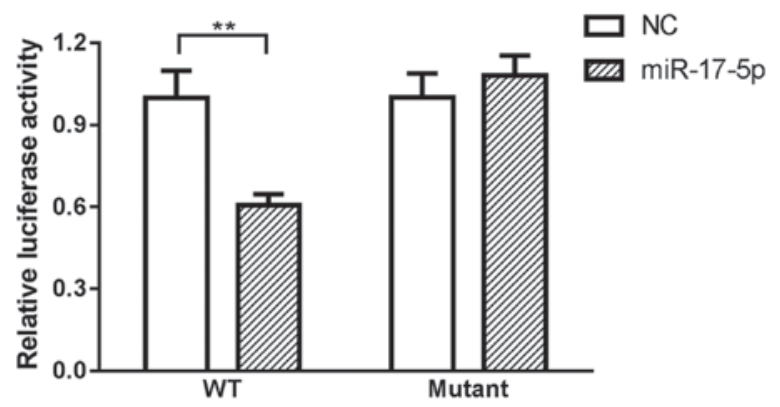

Figure 6. PTEN is direct target of miR-17-5p. (A) Sequence alignment of the paired 3'-UTR of miR-17-5p and PTEN by bioinformatics tool Targetscan and miRanda; (B) Results of dual luciferase reporter assay. ${ }^{* *} \mathrm{P}<0.01$. WT, Fragments of wild-type PTEN 3'-UTR; Mutant, fragments of wild-type PTEN 3'-UTR; NC, miR-17-5p negative control; PTEN, Phosphatase and tensin homolog; GAPDH, glyceraldehyde-3-phosphate dehydrogenase.

Next, we transfected HTMCs with either miR-17-5p mimics or miR-17-5p inhibitor and evaluated the effect of miR-17-5p on the proliferation and apoptosis of HTMCs. We observed that transient overexpression of miR-17-5p induced a significant increase in the proliferation and a significant decrease in the apoptosis of HTMCs $(\mathrm{P}<0.05)$, while knockdown of miR-17-5p exhibited the opposite results. Moreover, $\mathrm{Bcl}-2$ and Bcl-xL are known to be anti-apoptotic proteins, and Bax is a pro-apoptotic protein $(27,28)$. Our data indicated that transfection of miR-17-5p mimics promoted the expression of Bcl-2 and Bcl-xL, and suppressed the expression of Bax, while knockdown of miR-17-5p exhibited the opposite effects. Taken together, these results indicate that miR-17-5p can regulate the proliferation and apoptosis of HTMCs in vitro.

PTEN is known to be a tumor suppressor in the field of cancer studies (29). Knockdown of PTEN can lead to an increase in the proliferation and a decrease in the apoptosis rate of many cancer cell types, leading to the incidence and development of various diseases (30-32). However, in the case of glaucoma, PTEN may play a different role. It has been observed that increased expression of PTEN can lead to the degeneration of optic neurons, and knockdown of PTEN can significantly alleviate optic nerve damage in glaucoma. In a very recent study, Tellios et al (33) demonstrated that phosphorylation of PTEN is significantly increased in TMCs upon treatment with TGF- $\beta$ (increased levels of TGF- $\beta$ in the aqueous humor is one of the main causes of fibrosis in OAG), and an increase in the expression of PTEN may contribute to the fibrosis of the HTMCs in glaucoma. In the present study, we observed that the expression of PTEN was markedly increased in HTMCs following exposure to $\mathrm{H}_{2} \mathrm{O}_{2}(\mathrm{P}<0.01)$, suggesting that increased PTEN may be involved in the pathogenesis of glaucoma. Moreover, transfection of miR-17-5p mimic or inhibitor induced a significant decrease or increase in the expression of PTEN, respectively. The results from the dual luciferase reporter assay confirmed that PTEN is a direct target of miR-17-5p. In summary, these data indicated that miR-17-5p can regulate the proliferation and apoptosis of HTMCs by targeting PTEN.
The present study has certain limitations. We only performed cellular experiments, and the expression levels of miR-17-5p in tissue samples from patients and normal controls should be evaluated to confirm our findings from the clinical perspective. However, due to ethical issues, it is difficult to obtain the trabecular meshwork of healthy volunteers; thus, in vivo studies using animal models could be a better option.

In conclusion, our data demonstrated that miR-17-5p is down-regulated and PTEN is up-regulated in HTMCs under oxidative conditions. We also identified that miR-17-5p can regulate the proliferation and apoptosis of HTMCs through targeting PTEN. Our data indicated that miR-17-5p has the potential to become a novel therapeutic target for the treatment of glaucoma.

\section{Acknowledgements}

Not applicable.

\section{Funding}

The persent study was sponsored by the funds from the project of the Department of Health, Heilongjiang Province (project no. 2011-005).

\section{Availability of data and materials}

The datasets used and/or analyzed during the present study are available from the corresponding author on reasonable request.

\section{Authors' contributions}

FZ designed the study and wrote the majority of the manuscript. XW performed most of the experiments and wrote part of the manuscript. ZL and JB performed some of the experiments. WS performed some of the statistical analysis.

\section{Ethics approval and consent to participate}

Not applicable.

\section{Patient consent for publication}

Not applicable.

\section{Competing interests}

The authors declare that they have no competing interests.

\section{References}

1. Zhao R, Yin D, Wang E and Si B: The effect of MTHFR ala222val polymorphism on open-angle glaucoma: A meta-analysis. Ophthalmic Genet 36: 27-30, 2015.

2. Tham YC, Li X, Wong TY, Quigley HA, Aung T and Cheng CY: Global prevalence of glaucoma and projections of glaucoma burden through 2040: A systematic review and meta-analysis. Ophthalmology 121: 2081-2090, 2014.

3. Bailey JN, Loomis SJ, Kang JH, Allingham RR, Gharahkhani P, Khor CC, Burdon KP, Aschard H, Chasman DI, Igo RP Jr, et al: Genome-wide association analysis identifies TXNRD2, ATXN2 and FOXC1 as susceptibility loci for primary open-angle glaucoma. Nat Genet 48: 189-194, 2016. 
4. Liang YB, Wang NL, Rong SS and Thomas R: Initial treatment for primary angle-closure glaucoma in China. J Glaucoma 24: 469-473, 2015

5. Nongpiur ME, Khor CC, Jia H, Cornes BK, Chen LJ, Qiao C, Nair KS, Cheng CY, Xu L, George R, et al: ABCC5, a gene that influences the anterior chamber depth, is associated with primary angle closure glaucoma. PLoS Genet 10: e1004089, 2014.

6. Shen W, Han Y, Huang B, Qi Y, Xu L, Guo R, Wang X and Wang J: MicroRNA-483-3p inhibits extracellular matrix production by targeting Smad4 in human trabecular meshwork cells Invest Ophthalmol Vis Sci 56: 8419-8427, 2015

7. Xu L, Zhang Y, Guo R, Shen W, Qi Y, Wang Q, Guo Z, Qi C, Yin $\mathrm{H}$ and Wang J: HES1 promotes extracellular matrix protein expression and inhibits proliferation and migration in human trabecular meshwork cells under oxidative stress. Oncotarget 8: 21818-21833, 2017.

8. Feng R, Sang Q, Zhu Y, Fu W, Liu M, Xu Y, Shi H, Xu Y, Qu R, Chai R, et al: MiRNA-320 in the human follicular fluid is associated with embryo quality in vivo and affects mouse embryonic development in vitro. Sci Rep 5: 8689, 2015.

9. Li Y, Cai B, Shen L, Dong Y, Lu Q, Sun S, Liu S, Ma S, Ma PX and Chen J: MiRNA-29b suppresses tumor growth through simultaneously inhibiting angiogenesis and tumorigenesis by targeting Akt3. Cancer Lett 397: 111-119, 2017.

10. Qun L, Wenda X, Weihong S, Jianyang M, Wei C, Fangzhou L, Zhenyao X and Pingjin G: miRNA-27b modulates endothelial cell angiogenesis by directly targeting Naa15 in atherogenesis Atherosclerosis 254: 184-192, 2016.

11. Falzone L, Candido S, Salemi R, Basile MS, Scalisi A, McCubrey JA, Torino F, Signorelli SS, Montella M and Libra M: Computational identification of microRNAs associated to both epithelial to mesenchymal transition and NGAL/MMP-9 pathways in bladder cancer. Oncotarget 7: 72758-72766, 2016

12. Bagnoli M, De Cecco L, Granata A, Nicoletti R, Marchesi E, Alberti P, Valeri B, Libra M, Barbareschi M, Raspagliesi F, et al: Identification of a chrXq27.3 microRNA cluster associated with early relapse in advanced stage ovarian cancer patients. Oncotarget 2: 1265-1278, 2011.

13. Guo R, Shen W, Su C, Jiang S and Wang J: Relationship between the pathogenesis of glaucoma and miRNA. Ophthalmic Res 57: 194-199, 2017.

14. Molasy M, Walczak A, Szaflik J, Szaflik JP and Majsterek I: MicroRNAs in glaucoma and neurodegenerative diseases. J Hum Genet 62: 105-112, 2017.

15. Paylakhi SH, Yazdani S, April C, Fan JB, Moazzeni H, Ronaghi M and Elahi E: Non-housekeeping genes expressed in human trabecular meshwork cell cultures. Mol Vis 18: 241-254, 2012.

16. Tsuchida A, Ohno S, Wu W, Borjigin N, Fujita K, Aoki T, Ueda S, Takanashi M and Kuroda M: miR-92 is a key oncogenic component of the miR-17-92 cluster in colon cancer. Cancer Sci 102 2264-2271, 2011.

17. Zhu Y, Gu J, Li Y, Peng C, Shi M, Wang X, Wei G, Ge O, Wang D, Zhang B, et al: MiR-17-5p enhances pancreatic cancer proliferation by altering cell cycle profiles via disruption of RBL2/E2F4-repressing complexes. Cancer Lett 412: 59-68, 2018

18. Liao XH, Xiang Y, Yu CX, Li JP, Li H, Nie Q, Hu P, Zhou J and Zhang TC: STAT3 is required for MiR-17-5p-mediated sensitization to chemotherapy-induced apoptosis in breast cancer cells. Oncotarget 8: 15763-15774, 2017.

19. Jin T, Wu X, Yang H, Liu M, He Y, He X, Shi X, Wang F, Du S Ma Y, et al: Association of the miR-17-5p variants with susceptibility to cervical cancer in a Chinese population. Oncotarget 7 : $76647-76655,2016$.
20. Shi J, Bei Y,Kong X, Liu X, Lei Z, Xu T, Wang H, Xuan Q, Chen P, $\mathrm{Xu}$ J, et al: $\mathrm{miR}-17-3 \mathrm{p}$ contributes to exercise-induced cardiac growth and protects against myocardial ischemia-reperfusion injury. Theranostics 7: 664-676, 2017.

21. Tan L, Meng L, Shi X and Yu B: Knockdown of microRNA-17-5p ameliorates atherosclerotic lesions in ApoE-/- mice and restores the expression of very low density lipoprotein receptor. Biotechnol Lett 39: 967-976, 2017.

22. Li G, Luna C, Qiu J, Epstein DL and Gonzalez P: Alterations in microRNA expression in stress-induced cellular senescence. Mech Ageing Dev 130: 731-741, 2009.

23. Yu AL, Fuchshofer R, Kampik A and Welge-Lüssen U: Effects of oxidative stress in trabecular meshwork cells are reduced by prostaglandin analogues. Invest Ophthalmol Vis Sci 49: 4872-4880, 2008

24. Chen M, Liu B, Gao Q, Zhuo Y and Ge J: Mitochondria-targeted peptide MTP-131 alleviates mitochondrial dysfunction and oxidative damage in human trabecular meshwork cells. Invest Ophthalmol Vis Sci 52: 7027-7037, 2011.

25. Wang Y, Li F and Wang S: MicroRNA-93 is overexpressed and induces apoptosis in glaucoma trabecular meshwork cells. Mol Med Rep 14: 5746-5750, 2016.

26. Luna C, Li G, Qiu J, Epstein DL and Gonzalez P: MicroRNA-24 regulates the processing of latent TGF $\beta 1$ during cyclic mechanical stress in human trabecular meshwork cells through direct targeting of FURIN. J Cell Physiol 226: 1407-1414, 2011.

27. Reshi L, Wang HV, Hui CF, Su YC and Hong JR: Anti-apoptotic genes Bcl-2 and Bcl-xL overexpression can block iridovirus serine/threonine kinase-induced Bax/mitochondria-mediated cell death in GF-1 cells. Fish Shellfish Immunol 61: 120-129, 2017.

28. Yang $J$ and Yao S: JNK-Bcl-2/Bcl-xL-Bax/Bak pathway mediates the crosstalk between matrine-induced autophagy and apoptosis via interplay with Beclin 1. Int J Mol Sci 16: 25744-25758, 2015.

29. Liu H, Pan Y, Han X, Liu J and Li R: MicroRNA-216a promotes the metastasis and epithelial-mesenchymal transition of ovarian cancer by suppressing the PTEN/AKT pathway. Onco Targets Ther 10: 2701-2709, 2017.

30. Ronen S, Abbott DW, Kravtsov O, Abdelkader A, Xu Y, Banerjee A and Iczkowski KA: PTEN loss and p27 loss differ among morphologic patterns of prostate cancer, including cribriform. Hum Pathol 65: 85-91, 2017.

31. Feng Y, Zou W, Hu C, Li G, Zhou S, He Y, Ma F, Deng C and Sun L: Modulation of CASC2/miR-21/PTEN pathway sensitizes cervical cancer to cisplatin. Arch Biochem Biophys 623-624: 20-30, 2017.

32. Li S, Shen Y, Wang M, Yang J, Lv M, Li P, Chen Z and Yang J: Loss of PTEN expression in breast cancer: Association with clinicopathological characteristics and prognosis. Oncotarget 8: 32043-32054, 2017.

33. Tellios N, Belrose JC, Tokarewicz AC, Hutnik C, Liu H, Leask A, Motolko M, Iijima M and Parapuram SK: TGF- $\beta$ induces phosphorylation of phosphatase and tensin homolog: Implications for fibrosis of the trabecular meshwork tissue in glaucoma. Sci Rep 7: 812, 2017

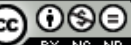

This work is licensed under a Creative Commons Attribution-NonCommercial-NoDerivatives 4.0 International (CC BY-NC-ND 4.0) License. 